\title{
PHOTONIC FRACTALS OF DIELECTRIC AND METAL MEDIA FOR ELECTROMAGNETIC WAVE LOCALIZATION
}

Y. Miyamoto and S. Kirihara

Smart Processing Research Center, Joining and Welding Research Institute

Osaka University, Ibaraki, Osaka 567-0047, Japan

K.Sakoda

Nanomaterials Laboratory, National

Institute for Materials Science

Tsukuba, Ibaraki 305-0003, Japan
M.W. Takeda and K.Honda

Faculty of Science, Shinshu University

Matsumoto, Nagano 390-8621, Japan

\begin{abstract}
3D photonic fractals with the self-similar structure can localize electromagnetic waves completely without reflection and transmission. Such fractals of the Menger sponge structure were successfully fabricated with dielectric and metal media using $\mathrm{CAD} / \mathrm{CAM}$ stereolithography. The localized frequency in dielectric Menger sponge structures is predicted using an empirical equation associated with the fractal geometry and spatially averaged dielectric constant, thus the frequency of the localized modes can be designed. For metal Menger sponge structures, the localized frequency could be determined mainly based on the geometrical factors. The localization mechanism in a photonic fractal is essentially different from the localization in a photonic crystal with the periodic structure. These new functionally structured materials of photonic fractals are considered to be applicable in a wide area of communication, information, energy, sensing, medical care, and others.
\end{abstract}

\section{INTRODUCTION}

Electromagnetic or optical wave interactions with periodic structures like photonic crystals have been studied intensively in the last two decades. Bragg scattering in photonic crystals creates the photonic band gap and localization of electromagnetic wave. ${ }^{1-3}$ It is known that the band gap is formed not only in dielectric photonic crystals, but also in metallic ones. ${ }^{4-5}$

To the extent authorized under the laws of the United States of America, all copyright interests in this publication are the property of The American Ceramic Society. Any duplication, reproduction, or republication of this publication or any part thereof, without the express written consent of The American Ceramic Society or fee paid to the Copyright Clearance Center, is prohibited. 
While, the wave interactions with fractal structures have attracted attention in recent years as well. Fractal structure is characterized with the self-similarity which means that the local configuration is similar to the whole configuration. ${ }^{6}$ It has no periodic symmetry. Such self-similar structures show interesting reflection and transmission phenomena. ${ }^{7-9}$, and strong localization of electromagnetic or optical waves. ${ }^{10}$ However, these studies were limited in fractal planes or multilayers with 1 or 2 dimensional geometry so that the complete localization in a three dimensional space has not been achieved.

Recently, we have fabricated 3D fractal structures called Menger sponge with epoxy or $\mathrm{TiO}_{2}-\mathrm{SiO}_{2}$ dispersed epoxy by $\mathrm{CAD} / \mathrm{CAM}$ stereolithography and found that the incident microwave with the specific wavelength is completely localized or confined without reflection and transmission. ${ }^{11-12}$ Because no such material was known that can localize electromagnetic waves three dimensionally without reflection and transmission, we named it as photonic fractal. Furthermore, we have found that metal Menger sponge structures can also localize electromagnetic waves. Such localization function of electromagnetic waves or energy is applicable in communication, information, energy, sensing, medical care, and other fields.

In the present paper we report on fabrication of such 3D fractal structures of dielectric and metal media, their electromagnetic wave responses, and examine in some detail the localization behaviors.

\section{GEOMETRY OF MENGAER SPONGE FRACTAL}

Menger sponge is a 3D version of the Cantor bar fractal. Cantor bar is formed by extracting a center segment of three equivalent segments divided from an initial bar and repeating this process to the remaining two side segments. When this operation is conducted one time, the resulting Cantor bar is called stage 1. By repeating the operation, the stage number increases as shown in Fig.1 (a). Figure 1 (b) is called Sierpinski's carpet which is a two dimensional version of Cantor bar. Its stage number is 3. Figure 1 (c) is a stage 3 Menger sponge, which is formed by dividing the initial cube into 27 identical cube pieces, and extracting seven pieces at the body- and face-centers. 


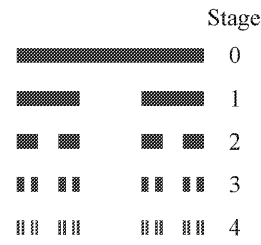

(a)

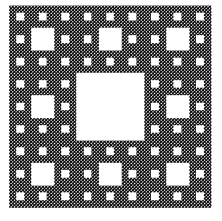

(b)

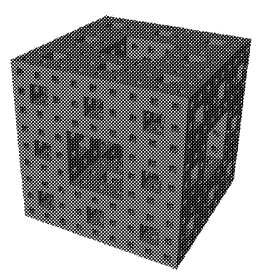

(c)

Figure 1. 1D, 2D, and 3D fractals; (a) Cantor bar, (b) Sierpinski's Carpet, (c) Menger sponge.

The fractal dimension $D$ is used as an important parameter to describe the complexity of fractal structures, which is calculated by the following equation:

$$
\mathrm{N}=\mathrm{S}^{D}
$$

where $\mathrm{N}$ is the number of the self-similar units newly created when the size of the initial unit decreases to $1 / \mathrm{S}$. In Cantor bar, $\mathrm{N}=2$ and $\mathrm{S}=3$, so that $\mathrm{D}=\log 2 / \log 3=0.63$, in Sierpinski's Carpet, $\mathrm{N}=8, \mathrm{~S}=3, \mathrm{D}=\log 8 / \log 3 \fallingdotseq 1.89$, in Menger sponge, $\mathrm{N}=20$, and $\mathrm{S}=3$, $D=\log 20 / \log 3=2.73$. It is noted that the fractal structure is characterized mathematically by the fractal dimension with decimal figures.

\section{EXPERIMENTAL}

We designed dielectric Menger sponge structures on a computer using a CAD program (Toyota Caelum Co. Ltd, thinkdesing ver.5.0). The designed structure is converted into a rapid prototyping format (STL file), sliced into a set of thin sections, and transferred to a stereographic machine (D-MEC Co.Ltd, Japan, SCS-300P). This machine forms a three-dimensional object layer-by-layer by scanning a UV laser of 355 $\mathrm{nm}$ in wavelength over a liquid photopolymer epoxy resin. ${ }^{13}$ The thickness of each layer is about $150 \mu \mathrm{m}$. The $\mathrm{TiO}_{2}-\mathrm{SiO}_{2}$ powders with a particle size of about $10 \mu \mathrm{m}$ are dispersed into the liquid resin in order to increase the dielectric constant of the fractal structure.

Metallic Menger sponge structures were fabricated by vacuum casting the molten Bi-Pb-Sn-In eutectic alloy with melting point at $70^{\circ} \mathrm{C}$ into the epoxy mold, which was 
formed by stereolithography. The epoxy mold was removed in acetone after casting.

Microwave responses of these Menger sponge structures were measured using network analyzer (Agilent Technologies, E8364B ) and two horn antennas as shown in Fig.2 (a). The measured frequency range was $6 \mathrm{GHz}-20 \mathrm{GHz}$. The electric field profile at the inner and outer air space of Menger sponges was mapped by using a monopole antenna against the microwave at the localized frequency emitted from a horn antenna as shown in Fig.2(b). The dielectric constant of the $\mathrm{TiO}_{2}-\mathrm{SiO}_{2}$ dispersed epoxy was measured by using dielectric kit ( Agilent Technologies, HP85070B ).

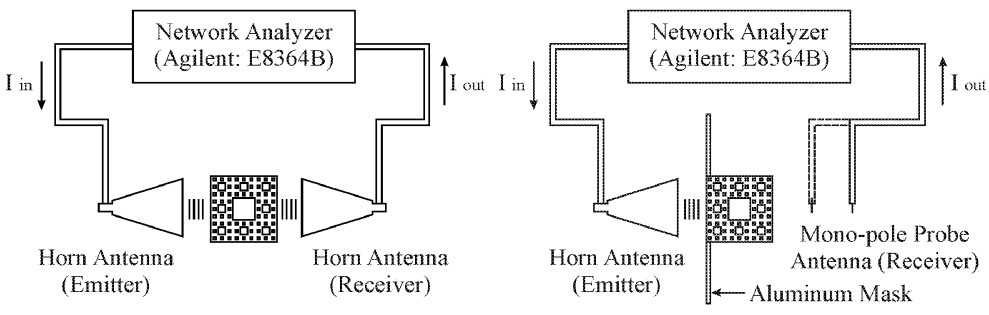

Attenuation: $\mathrm{dB}=10 \log (\mathrm{I}$ out $/ \mathrm{I}$ in $)$

(a)
Electric Field Intensity: $\mathrm{dB}=10 \log (\mathrm{I}$ out $/ \mathrm{I}$ in $)$

(b)

Figure 2. Measurement systems of microwave responses, (a) Reflection and transmission measurement in a free space, (b) Electric field measurement at the inner and outer air space of Menger sponge structure.

\section{RESULTS AND DISCUSSION}

Localization in Dielectric Menger Sponge Fractals

Figure 3 shows a stage 3 Manger sponge structure with an edge length of $81 \mathrm{~mm}$, which was formed of $10 \mathrm{vol} . \% \mathrm{TiO}_{2}-\mathrm{SiO}_{2}$ dispersed epoxy and the transmission and reflection spectra against normally incident microwaves in free space. Both sharp attenuations for reflection and transmission to $-60 \mathrm{~dB}$ were observed at $12.0 \mathrm{GHz}$ suggesting the strong localization without reflection and transmission.

Figure 4 shows a profile of the electric field intensity in the inner and outer air space of the stage 3 Menger sponge at the central cross section in x-y plane, which was measured using a monopole probe antenna against the incident microwave at $12.0 \mathrm{GHz}$ emitted from a horn antenna. The field intensity was most concentrated in the central air cube. Similar electric field profiles were obtained for other cross sectional planes and 
for the stage 4 Menger sponge with the same size as the stage 3 Menger sponge.
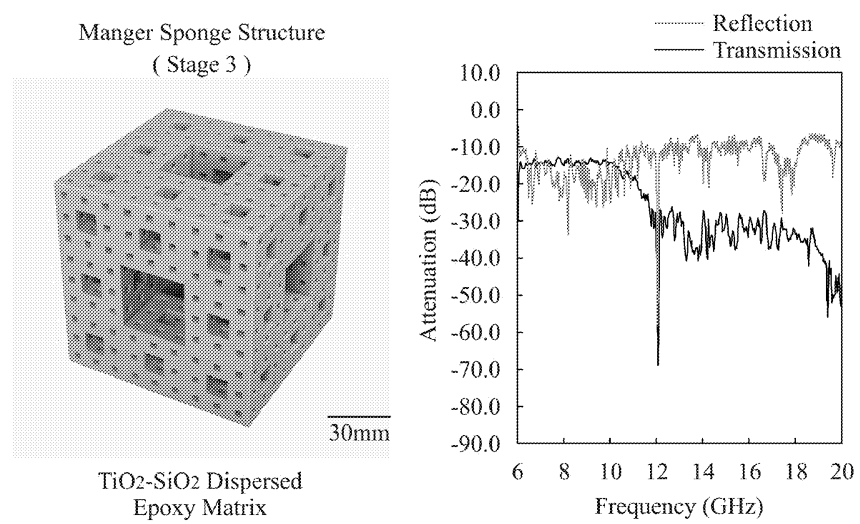

Figure 3. Photo of a stage $3 \mathrm{Menger}$ sponge structure of $\mathrm{TiO}_{2}-\mathrm{SiO}_{2} /$ epoxy with $81 \mathrm{~mm}$ cube, and the reflection and transmission spectra.

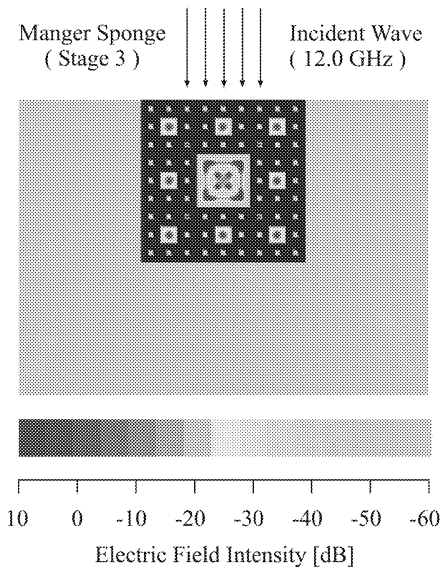

Figure 4. Profile of electric field intensity at the cross sectional $x-y$ plane in a stage 3 Menger sponge structure of $\mathrm{TiO}_{2}-\mathrm{SiO}_{2} /$ epoxy with $81 \mathrm{~mm}$ cube.

However, the electric field leaked out of the stage 2 Menger sponge though the resonated field intensity was observed in the air cubes as seen in Fig.5. Figure 6 shows a 
stage 4 Manger sponge with an edge length of $81 \mathrm{~mm}$ and the transmission and reflection spectra. Sharp attenuations for both reflection and transmission to $-70 \mathrm{~dB}$ were observed at $13.6 \mathrm{GHz}$. These results suggest that the strong localization of microwaves occurs in dielectric Menger sponge structures with the stage numbers higher than 3.

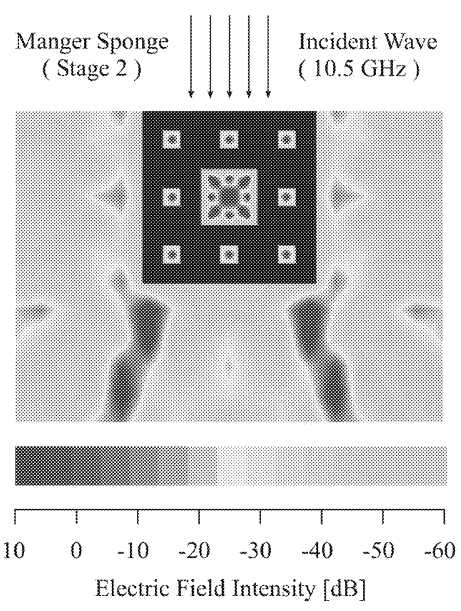

Figure 5. Profile of electric field intensity at the cross sectional x-y plane in a stage 2 Menger sponge structure of $\mathrm{TiO}_{2}-\mathrm{SiO}_{2} /$ epoxy with $81 \mathrm{~mm}$ cube.
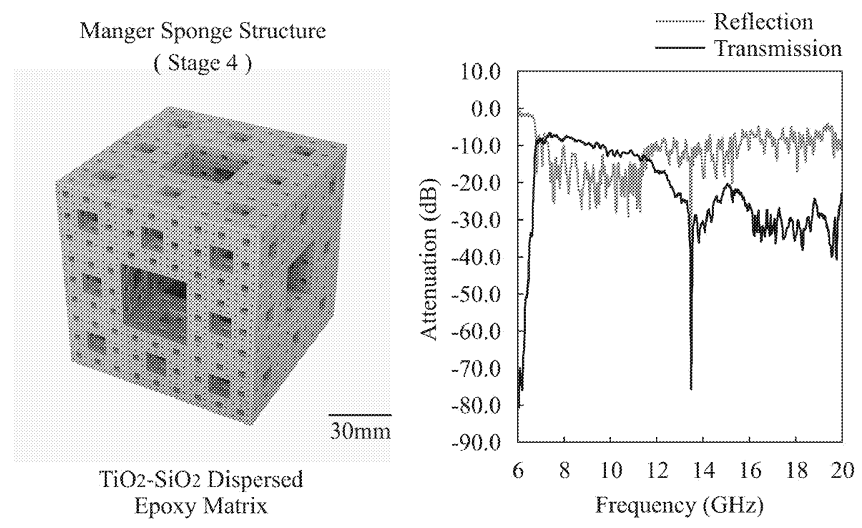

Figure 6. Photo of a stage 4 Menger sponge structure of $\mathrm{TiO}_{2}-\mathrm{SiO}_{2} /$ epoxy with $81 \mathrm{~mm}$ 
cube, and the reflection and transmission spectra.

The localization of electromagnetic waves in dielectric Menger sponges can not be explained by Bragg scattering and the band gap produced in periodic structures like photonic crystals. Probably such localization would occur due to the self-similar resonances of multiply reflected or scatted waves in a 3D fractal structure. Though the localization mechanism of electromagnetic waves in these Menger sponge fractals is not well understood yet, an empirical equation could be derived to predict the frequency of the localized mode in dielectric Menger sponges as follows.

$$
\lambda_{\text {conf }}=2^{\ell} a \sqrt{ } \varepsilon_{\text {eff }} / 3^{2 \ell-1}
$$

where $\lambda$ conf is the wavelength of the localized mode in air. lis the order number of the localized modes. The effective dielectric constant $\varepsilon$ eff is the spatially averaged dielectric constant of the Menger sponge structure. The filling factor $f$ is the volumetric ratio of remaining parts in an $\mathrm{n}$-dimensional fractal. In the case of the stage $\mathrm{m}$ Menger sponge, it is expressed by $f=\left(\mathrm{N} / \mathrm{S}^{\mathrm{n}}\right)^{\mathrm{m}}$. Thus, the filling factor of dielectric medium in the stage 3 Menger sponge is $(20 / 27)^{3}$. The effective dielectric constant $\varepsilon_{\text {eff }}$ is given by the equation of $\varepsilon_{\text {eff }}=f \varepsilon_{A}+(1-f) \varepsilon_{B}$, where $\varepsilon_{A}$ and $\varepsilon_{B}$ are dielectric constants of remaining and extracted parts, respectively. The dielectric constant of the bulk epoxy including $10 \mathrm{vol} . \% \mathrm{TiO}_{2}-\mathrm{SiO}_{2}$ measured was $\varepsilon_{\mathrm{A}}=8.8 . \quad \varepsilon_{\mathrm{B}}=1$ (air). Therefore, the effective dielectric constant for the stage 3 Menger sponge is calculated as 4.2.

Table I compares the calculated and measured frequencies of localization in the dielectric Menger sponges with different sizes, stages, and dielectric constants. Other data for stage 3 Menger sponges (27mm cube) of epoxy and $\mathrm{TiO}_{2}-\mathrm{SiO}_{2} /$ epoxy, which were obtained in our previous study, are added in the table. ${ }^{11-12}$ In these smaller Menger sponges, the localized frequencies measured at $12.8 \mathrm{GHz}$ for the epoxy and $8.0 \mathrm{GHz}$ for the $\mathrm{TiO}_{2}-\mathrm{SiO}_{2}$ /epoxy show good agreements with the calculated ones as the first localized mode $(l=1)$.

Table I. Measured and calculated frequencies of localized modes in various Menger sponge structures.

\begin{tabular}{cccccc}
\hline \hline $\begin{array}{c}\text { Cube Size; } \\
\text { a }\end{array}$ & Stage & Effective & Calculated & Calculated & Measured \\
& Number; & Dielectric & Localized & Localized & Localized \\
{$[\mathrm{mm}]$} & $\mathrm{m}$ & Constant; & Frequency & Frequency & Frequency \\
& & $\varepsilon_{\text {eff }}$ & $(\ell=1)[\mathrm{GHz}]$ & $(\ell=2)[\mathrm{GHz}]$ & {$[\mathrm{GHz}]$} \\
\hline
\end{tabular}




\begin{tabular}{cccccc}
\hline 27 & 3 & 1.7 & 12.8 & 57.5 & $12.8^{11}$ \\
27 & 3 & 4.2 & 8.1 & 36.6 & $8.0^{12}$ \\
81 & 3 & 4.2 & 2.7 & 12.2 & 12.0 \\
81 & 4 & 3.3 & 3.1 & 13.8 & 13.6 \\
\hline
\end{tabular}

The calculated frequencies for the second modes $(\ell=2)$ were $57.5 \mathrm{GHz}$ and $36.6 \mathrm{GHz}$, respectively. These frequencies were high and out of measurable range in our measurement system.

For the present stage 3 and 4 Menger sponges $(81 \mathrm{~mm}$ cube), the measured frequencies were $12.0 \mathrm{GHz}$ and $13.6 \mathrm{GHz}$, respectively. These values agree well with the calculated ones for the second modes. The calculated frequencies for the first modes were out of measurable range.

In a dielectric medium with the dielectric constant $\varepsilon$, the wavelength of

electromagnetic wave is reduced to $1 / \sqrt{ } \varepsilon$. Equation (2) reveals that the wavelength of the localized mode in a dielectric Menger sponge structure corresponds to $2 / 3$ of its edge length for the first mode and $4 / 9$ for the second mode. This means that the strong localization can occur in such a narrow space with the size of 1.5 or 2 times of the wavelength in dielectric Menger sponges with the stage number greater than 3 . It is very efficient comparing with photonic crystals, which need a space of periodic structure with several times of wavelength for the strong localization.

\section{Localization in Metal Menger Sponge Fractals}

Figure 7 shows a stage 3 metal Menger sponge fractal with an edge length of $81 \mathrm{~mm}$ and the transmission and reflection spectra against normally incident microwaves. The metal Menger sponge was well fabricated without distortions, cracks, and pores. Very sharp attenuations of transmission and reflection down to $-80 \mathrm{~dB}$ appeared at $15.1 \mathrm{GHz}$. The strong intensity profile of the electric field at $15.1 \mathrm{GHz}$ was observed in the central air cube as shown in Fig.8. Stage 2 and 4 metal Menger sponges with the same size as the stage 3 showed more shallow and deep dips without leaking of incident microwave at the same $15.1 \mathrm{GHz}$, respectively, however, the stage 1 sample showed no localization.

P. Sheng and his co-workers reported on anomalous behaviors in reflectance and transmittance of microwaves for two dimensional $\mathrm{Cu}$ metal fractals. ${ }^{8-9}$ While, Shalaef's group found extremely strong localizations of light at random fractal films consisting of nano-sized silver particles aggregate. ${ }^{10}$ However, in these $2 \mathrm{D}$ fractal structures, it is impossible to completely localize the incident microwaves or light. 
In the case of 3D dielectric Menger sponge fractals, the localized wavelength and frequency depended on the effective dielectric constant, which changes with the stage number even though the size and material are the same. While the metal Menger sponges showed no dependence of the localized frequency on the stage numbers. The dielectric constant of metal is negative so that electromagnetic waves can not penetrate into metal and scatter at the surface. Figure 7 shows the existence of the cut-off frequency at about $6 \mathrm{GHz}$. It corresponds to the wavelength of $50 \mathrm{~mm}$. The edge length of the largest central air cube is $27 \mathrm{~mm}$ near the half wavelength, while, the wavelength of the localized mode at $15.1 \mathrm{GHz}$ is about $20 \mathrm{~mm}$.

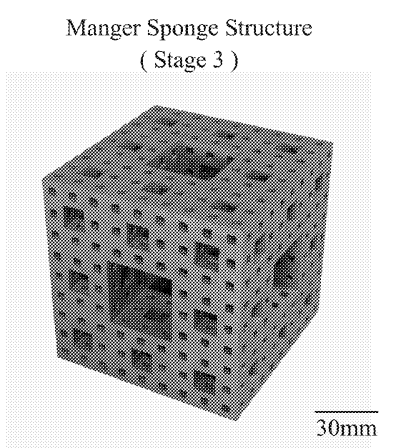

Bi-Sn-Pb-In Eutectic Alloy Matrix

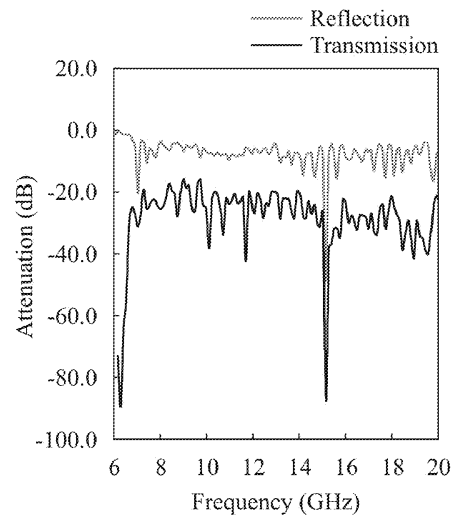

Figure 7. Photo of a stage 3 Menger sponge structure of Bi-Sn-Pb-In eutectic alloy with $81 \mathrm{~mm}$ cube, and the reflection and transmission spectra. 


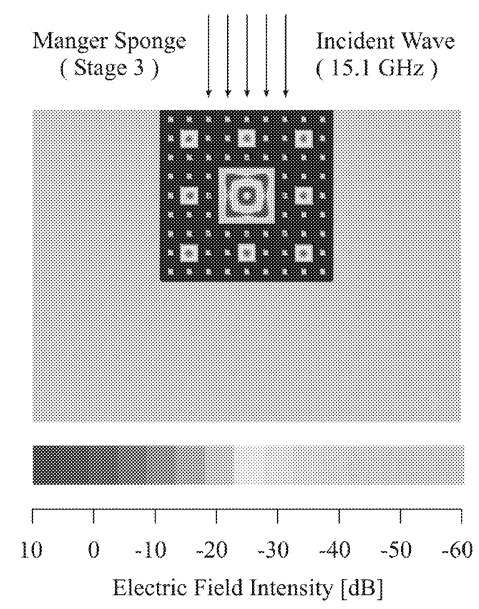

Figure 8. Profile of electric field intensity at the cross sectional x-y plane in a stage 3 Menger sponge structure of Bi-Sn-Pb-In eutectic alloy with $81 \mathrm{~mm}$ cube.

The $3 / 2$ and $1 / 2$ wavelengths correspond nearly to each edge length of the center $(27 \mathrm{~mm})$ and second air cubes $(9 \mathrm{~mm})$, respectively. It is considered, therefore, that the measured localized mode may exist mainly in the central and second air cubes. The electric field intensity profile measured supports this estimation.

At present, we can not explain why electromagnetic waves localize in these dielectric and metal Menger sponge fractals, but the 3D fractal structure should play an essential role for the complete localization of waves. The theoretical study on the localization mechanism will be reported elsewhere. ${ }^{14}$

\section{POTENTIAL APPLICATIONS OF PHOTONIC FRACTAL}

The localization function of electromagnetic waves in 3D dielectric or metal fractal structures is applicable to various fields of information, communication, energy, medical care, and others. Fractal structures such as Menger sponge can provide an ideal absorber without reflectance and transmission for electromagnetic waves. Of course, when the dielectric fractal structure is made in optical wave scales, the light can be localized and confined that will be used for many photonic applications. It may be possible to localize and accumulate the electromagnetic energy in dielectric or metal fractal structures, if the structure is composed of an efficiently low loss material and the number of stage $m$ is sufficient which can reduce the medium of fractal. Such energy 
accumulation in the 3D fractal structure may provide new heat treatment devices for industrial, home and medical uses depending on the power. If high efficiency in energy accumulation could be realized by choosing ceramic, metal or their composite materials, new functional devices of photonic energy storage, capacitor, and collector may be produced.

\section{CONCLUSION}

3D dielectric and metal photonic fractals of Menger sponge structure were successfully fabricated by using CAD/CAM stereolithography and their electromagnetic responses were measured. The obtained results can be summarized as follows.

1. Dielectric and metal Menger sponge structures with the stage number over 3 can localize strongly electromagnetic waves with the specific wavelength.

2. The wavelength and frequency of the localized modes in dielectric Menger sponges can be predicted using an empirical equation associated with the fractal geometry and spatially averaged dielectric constant, thus the frequency of the localized modes can be designed. For metal Menger sponges, the localized frequency may be determined mainly by the geometrical factors.

3. The localization mechanism in these dielectric and metal fractals is not well understood yet, however, the 3D fractal structure of Menger sponge would play an essential role for the localization.

4. Photonic fractals exhibit great potential for applications in information, communication, energy, medical and other various fields.

\section{ACKNOWLEDGEMENT}

This work is partially supported by the grant for the $21^{\text {st }}$ Century's COE program "Center of Excellence for Advanced Structural and Functional Materials Design" under the auspice of the Ministry of Education, Culture, Sports, Science and Technology, Japan.

\section{REFERENCES}

${ }^{1}$ E. Yablonovitch, "Inhibited Spontaneous Emission in Solid-State Physics and Electronics”, Phys. Rev.Lett., 58, 2059-62 (1987).

2 J.D.Joannopoulos, R.D.Maeda and J.N.winn, "Photonic Crystals" ( Princeton University Press, USA 1995)

${ }^{3}$ K.Inoue and K.Ohtaka (eds.),"Photonic Crystals-Physics, Fabrication and Applications", (Springer, Germany 2004). 
${ }^{4}$ M.M.Sigalas, R.Biswas, K.M.Ho and C.M.Soukoulis, D.D.Crouch, "Waveguides in three-dimensional metallic photonic band-gap materials", Phys Rev. B. 60, 4426-29 (1999).

5 J.G.Fleming, S.Y.Lin, I.El-Kady, R.Biswas and K.M.Ho, "All-metallic three-dimensional photonic crystals with a large infrared bandgap," Nature, 417, 52-55 (2002).

${ }^{6}$ B. B. Mandelbrot, "The Fractal Geometry of Nature" (W. H. Freeman \& Company, San Francisco, CA, 1982).

${ }^{7}$ X.Sun and D.L.Jaggard,"Wave interactions with generalized Cantor bar fractal multilayers", J.Appl. Phys., 70. 2500-07 (1991).

${ }^{8}$ W. Wen, L. Zhou, J. Li, W. Ge, C. T. Chen and P. Sheng" Subwavelength Photonic Band Gaps from Planar Fractals", Phys. Rev. Lett. 89, 223901-1-4 (2002).

${ }^{9}$ W.Wen, Z.Yang, G.Xu, Y.Chen, L.Zhou, W.Ge, C.T.Chan and P.Sheng, "Infrared passbands from fractal slit patterns on a metal plate", Appl. Phys. Lett., 83, 2106-08 (2003).

10 V.M.Shalaev" Nonlinear Optics of Random media: Fractal Composites and Metal-Dielectric Films" (Springer Tracts in Modern Physics 158, Springer, Berlin, Heidelberg, 2000).

${ }^{11}$ M.W.Takeda, S. Kirihara, Y.Miyamoto, K.Sakoda and K.Honda, Localization of electromagnetic waves in three-dimensional fractal cavities, Phys. Rev. Lett. 92,093902-1-4 (2004).

12 Y.Miyamoto, S.Kirihara, S.Kanehira, M.W.Takeda, K.Honda and K.Sakoda, Smart Processing Development of Photonic Crystals and Fractals, Int. J. Appl. Ceram.Technol. 1, 40-48 (2004).

${ }^{13}$ S.Kirihara, Y.Miyamoto, K.Yakenaga, M.W.Takeda and K.Kajiyama, "Fabrication of electromagnetic crystals with a complete diamond structure by stereolithography", Solid State Comm. 121, 435-39 (2002).

${ }^{14}$ K.Sakoda, S.Kirihara, Y.Miyamoto, M.W.Takeda and K.Honda, "Electromagnetic Eigenmodes of a Three-Dimensional Photonic Fractal", Phys. Rev. B., to have been submitted. 